\title{
Polarimetry-Based Distributed Scatterer Processing Method for PSI Applications
}

\author{
Adugna G. Mullissa ${ }^{\circledR}$, Daniele Perissin ${ }^{\circledR}$, Valentyn A. Tolpekin, Member, IEEE, and Alfred Stein
}

\begin{abstract}
Permanent scatterer interferometry is a multitemporal interferometric synthetic aperture radar technique that produces high-accuracy ground deformation measurement. A high density of permanent scatterer (PS) is required to provide accurate results. In natural environments with low PS density, distributed scatterers (DSs) could serve as additional coherent observations. This paper introduces a polarimetric scattering property-based adaptive filtering method that preserves PS candidates and filters DS candidates. To further increase the coherence estimate of DS candidates, the technique includes a complex coherence decomposition that adaptively selects the most stable scattering mechanisms, thus improving pixel coherence estimation. The proposed method was evaluated on 11 quad-polarized ALOS PALSAR images and 21 dual-polarized Sentinel-1 images acquired over San Fernando Valley, CA, USA, and Groningen, The Netherlands, respectively. The application of this method increased the number of coherent pixels by almost a factor of eight compared with a single-polarization channel. This paper concludes that a coherence estimate can be significantly improved by applying scattering property-based adaptive filtering and coherence matrix decomposition and accurate displacement measurements can be achieved.
\end{abstract}

Index Terms-Adaptive filtering, distributed scatterers (DSs), multitemporal interferometric synthetic aperture radar (InSAR), permanent scatterer interferometry (PSI), polarimetric optimization, polarimetric synthetic aperture radar interferometry.

\section{INTRODUCTION}

$\mathbf{M}$ ULTITEMPORAL interferometric synthetic aperture radar (InSAR) is a well-studied Earth observation technique that provides a millimeter-scale accuracy in ground deformation measurements [1], [6], [8], [14], [27]. It aims to identify stable radar targets that are strong deterministic scatterers and exploits their differential phase stability to measure ground displacement. To derive accurate model fitting for displacement measurement, a dense network of permanent scatterer (PS) candidates is required. Hence, multitemporal InSAR works well in urban areas, where scattering from buildings and other man-made structures guarantees a dense

Manuscript received May 5, 2017; revised September 13, 2017 and January 18, 2018; accepted January 23, 2018. (Corresponding author: Adugna G. Mullissa.)

A. G. Mullissa, V. A. Tolpekin, and A. Stein are with the Department of Earth Observation Science, Faculty of Geoinformation Science and Earth Observation, University of Twente, 7514AE Enschede, The Netherlands (e-mail: a.mullissa@utwente.nl; v.a.tolpekin@utwente.nl; a.stein@utwente.nl).

D. Perissin is with the Lyle School of Civil Engineering, Purdue University, West Lafayette, IN 47907 USA (e-mail: perissin@purdue.edu).

Color versions of one or more of the figures in this paper are available online at http://ieeexplore.ieee.org.

Digital Object Identifier 10.1109/TGRS.2018.2798705 network. However, it has achieved limited success in obtaining dense PS candidates in natural environments.

To overcome this limitation, extended radar targets, termed distributed scatterers (DSs), were exploited to obtain dense pixel candidates. These targets provide moderate coherence as they are affected by temporal and baseline decorrelation. By applying adaptive spatial filtering techniques, the signalto-noise ratio (SNR) for these targets may be improved. In previous works, different statistical methods were used to distinguish PS and DS candidates. Reference [9] used the Kolmogorov-Smirnov test to evaluate the similarity of the amplitude distribution in two image pixels, and [12] applied the Anderson-Darling test statistic. Reference [22] applied a robust t-test to improve the effectiveness of identifying statistical homogenous pixels (SHPs). With the availability of fully polarimetric data, [25] applied a likelihood ratio test to establish the similarity of two Wishart matrices in two pixels. To compute the interferometric coherence, pixels are categorized into SHP on which spatial averaging is performed. Reference [27] selected DS candidates by applying weights to interferograms to identify DS that are coherent in portions of the interferogram stack. This method relaxed restrictions imposed by the permanent scatterer interferometry (PSI) technique is proposed in [8].

Combined PS and DS interferometry, however, is effective if similar multiple scattering mechanisms are present in the resolution cell. In practice, when using medium-resolution SAR data, different DS scattering mechanisms are mixed both in mixed rural-urban areas and rural areas. Even though the SNR is improved by multilooking, the interference between the mixed scattering mechanisms decreases the overall coherence estimate in the resolution cell. This has important implications in deformation measurements when some of these scattering mechanisms are stable and some are not. Reference [11] introduced the Component extrAction and sElection SAR method that is based on using a principal component analysis to decompose the covariance matrix to mitigate the effects of layover in urban areas. This method is able to reduce the effects of decorrelation in the DS pixels. Recently, [2] applied an eigendecomposition on the coherence matrix to obtain the dominant scattering mechanism within DS pixels. This method is effective in reducing decorrelation by selecting the dominant scattering mechanism to derive the interferometric phases. However, it assumes that all secondary scattering mechanisms may constitute noise.

In this paper, polarimetric scattering mechanism-based adaptive spatial filtering is introduced to filter DS candidates. 
The coherence interferogram decomposition method [2] is modified to adaptively select secondary scattering mechanisms that interfere constructively with the dominant scattering mechanism. This method is applied in quasi-permanent scatterer (QPS) interferometry [27]. The objective of this paper is to adaptively filter DS candidates and select the most stable scattering mechanisms in the DS cell. In doing so, we aim to improve the pixel coherence estimate in a mixed urban-rural scene.

This paper is organized as follows. The methodology employed in this paper is described in Section II. The data sets used in this paper in Section III. Results obtained from the methods are shown in Section IV. Discussions and conclusions on the obtained results are presented in Section V.

\section{METHOD}

\section{A. SAR Polarimetry}

Fully polarimetric SAR sensors measure the scattering matrix $S$, which can be presented as

$$
S=\left[\begin{array}{ll}
S_{\mathrm{HH}} & S_{\mathrm{HV}} \\
S_{\mathrm{VH}} & S_{\mathrm{VV}}
\end{array}\right]
$$

where the complex scattering coefficient $S_{X Y}$ indexed as $X, Y=(H, V)$ represents the horizontal $(H)$ and vertical $(V)$ polarization states. In SAR polarimetry, this scattering matrix is represented by the target scattering vector $k$. Vectorization expands the scattering matrix using simpler canonical scattering mechanisms. The Pauli target scattering vector of a given scattering matrix in the monostatic case assuming reciprocity, i.e., $S_{\mathrm{HV}}=S_{\mathrm{VH}}$, is given as [17]

$$
k=\frac{1}{\sqrt{2}}\left[\begin{array}{lll}
S_{\mathrm{HH}}+S_{\mathrm{VV}} & S_{\mathrm{HH}}-S_{\mathrm{VV}} & 2 S_{\mathrm{VH}}
\end{array}\right]^{T}
$$

where ${ }^{T}$ designates a matrix transpose. In dual-polarimetric data as in Sentinel-1 configuration (VH VV), $k$ reduces to

$$
k=\left[\begin{array}{ll}
S_{\mathrm{VV}} & 2 S_{\mathrm{VH}}
\end{array}\right]^{T} .
$$

For deterministic point scatterers, $k$ describes the scattering process completely. For DSs, however, $k$ displays a random property depending on the condition that the SAR wavelength is smaller than the resolution cell. Hence, $k$ is modeled by a multivariate complex circular Gaussian probability density function [18]. The second-order statistics represented by the coherency matrix $T$ that completely define the randomness of $k$ are computed by assuming stationarity and ergodicity given as

$$
T=\left\langle k_{i} k_{i}^{\dagger}\right\rangle=\frac{1}{n} \sum_{i=1}^{n} k_{i} k_{i}^{\dagger}
$$

where ${ }^{\dagger}$ is the conjugate transpose, \langle\rangle is spatial or temporal average of pixels, and $n$ is the number of samples or looks used to estimate $T$. In this paper, stationarity and ergodicity are assumed in time so $n$ represents the number of images in the stack. Notice that no spatial averaging is applied, since $n$ is obtained from the temporal dimension. The polarimetric coherency matrix $T$ contains only polarimetric information. $T$ is modeled by the complex Wishart probability density function [13].

\section{B. Target Decomposition}

Target decomposition theorems are used to determine the dominant scattering mechanism present in the PolSAR data. The Cloude-Pottier eigenvalue-eigenvector decomposition method [5] breaks down $T$ into a sum of elementary scattering contributions. $T$ is a Hermitian positive semidefinite matrix, and hence, it can be factorized into a matrix of eigenvalues and eigenvectors. Thus

$$
T=\sum_{i=1}^{3} \sigma_{i} u_{i} u_{i}^{\dagger}
$$

where $u_{i}$ is the eigenvector and $\sigma_{i}$ is the eigenvalue. The eigenvector can be further expanded by using physical parameters of targets, i.e.,

$$
u_{i}=\left[\begin{array}{lll}
\cos \alpha_{i} & e^{j \delta_{i}} \sin \alpha_{i} \cos \beta_{i} & e^{j \psi_{i}} \sin \alpha_{i} \sin \beta_{i}
\end{array}\right]^{T}
$$

where $\alpha$ refers to the physical scattering mechanism as described in [5], $\beta$ refers to the orientation of the target within the RADAR line of sight, and $\delta$ and $\psi$ are the copolar and cross-polar phase angles, respectively. The average physical scattering mechanism $\tilde{\alpha}$ is obtained as

$$
\tilde{\alpha}=\sum_{i=1}^{3} P_{i} \alpha_{i}, \quad \text { where } P_{i}=\frac{\sigma_{i}}{\sum_{i=1}^{3} \sigma_{i}}
$$

where $P_{i}$ is the probability obtained from the eigenvalues $(\sigma)$. To describe the statistical disorder of the scattering mechanisms, the scattering entropy is used, given as

$$
H=-\sum_{i=1}^{3} P_{i} \log _{3} P_{i} .
$$

In the Cloude-Pottier target decomposition, $\tilde{\alpha}$ typically assumes a value between $0^{\circ}$ and $90^{\circ}$ and $H$ assumes a value between 0 and 1. $\tilde{\alpha}$ values that are close to $0^{\circ}$ resemble surface scattering, whereas $\tilde{\alpha}=45^{\circ}$ resembles a volume scatterer and $\tilde{\alpha}=90^{\circ}$ indicates a double-bounce scattering mechanism. $H$ values close to 0 represent a deterministic scattering mechanism and an $H$ value close to one represents a random scattering, where there is no dominant scattering mechanism. However, $\tilde{\alpha}$ by itself is not enough to define the scattering mechanisms, and hence, the different scattering mechanisms are described in the $H / \tilde{\alpha}$ plane [19].

\section{Homogenous Pixel Selection}

To preserve PS candidates in multitemporal InSAR processing and filter DS candidates, homogenous pixel patches should be identified. In this paper, similarity between the objective pixel to be filtered and its neighboring pixels within a neighborhood window is established based on similar scattering properties within spatially interconnected pixels.

The Cloude-Pottier eigenvalue-eigenvector decomposition [5] provides the scattering properties, i.e., average scattering mechanism $(\tilde{\alpha})$ and scattering entropy $(H)$ in each resolution cell. We derive the neighborhood similarity by classifying the scattering mechanisms. This is accomplished by implementing the Wishart distance measure [19] between 
the pixel's $T$ estimate and the coherence matrix of the $m$ th scattering mechanism class, $V_{m}$. Here, $V_{m}=E[T]$ for $T$ computed on $\omega_{m}$, that is, the pixels belonging to the $m$ th scattering mechanism class. The distance measured between pixel $T$ and $V_{m}$ is given as

$$
d\left(T, V_{m}\right)=\ln \left|V_{m}\right|+\operatorname{Tr}\left(V_{m}^{-1} T\right) .
$$

Here, $\omega_{m}$ is the pixel belonging to the $m$ th scattering mechanism class. The classification is implemented using an iterative combination of the unsupervised target decomposition classifier and the supervised Wishart classifier [19]. The initial scattering mechanism class was derived for each pixel by taking the zone center in the $H / \tilde{\alpha}$ scatter plot [5]. For dual-polarized data, the $H / \tilde{\alpha}$ zone is modified, as discussed in [16]. In subsequent classifications, the average $T$ from each scattering class defined in the $H / \tilde{\alpha}$ plane is used to define the new class coherence matrix $V_{m}$, and each pixel in the image is classified based on the new $V_{m}$ value. This process is repeated until a termination criterion is reached. In this paper, the number of pixels switching class when compared with a previous classification result is used.

The SHPs are defined within a $p \times p$ pixel window. We first discard isolated pixels that do not belong to a scattering class of the objective pixel to be filtered that is not spatially connected directly or through other SHPs. The spatially adaptive filtering is defined for pixel $W$ and all SHPs connected to pixel $W$. The brightest pixels that belong to the deterministic single-bounce or double-bounce class are left out as these may be PS candidates. This is accomplished by using the concept utilized in the Lee sigma filter [21]. We first calculate the 98th percentile of the double-bounce class obtained from the temporal average of $T_{22}$ intensity image [ $(1 / n) \sum_{i=1}^{n} \mid S_{\mathrm{HH}_{i}}-$ $\left.\left.S_{\mathrm{VV}_{i}}\right|^{2}\right]$ and single-bounce class obtained from the temporal average of $T_{11}$ intensity image $\left[(1 / n) \sum_{i=1}^{n}\left|S_{\mathrm{HH}_{i}}+S_{\mathrm{VV}_{i}}\right|^{2}\right]$. The 98th percentile and a threshold of 5 pixels within a window of $3 \times 3$ is used to determine if the target is a strong scatterer. Once the PS and DS patches are identified, adaptive spatial filtering of the objective pixel is performed within a $p \times p$ pixel window on the DS candidates only. The term spatial adaptive filtering mentioned throughout this paper refers to the selective complex averaging of pixels based on the identified SHPs. It includes the generation of SHPs by using scattering properties and space adaptive averaging of DS pixels. The methodology flowchart for the generation of SHPs is summarized in Fig. 1.

\section{Polarimetric Optimization}

Polarimetric optimization is a method applied to increase the differential phase quality and number of PS and DS involved in PSI by selecting the polarization state that is least affected by decorrelation [15], [24], [26]. To this effect, we first define the polarimetric interferometric coherency matrix $Z$, containing both polarimetric and interferometric information. $Z$ is given as

$$
Z=\left\langle K K^{\dagger}\right\rangle=\left[\begin{array}{cc}
T_{i i} & \Omega_{i j} \\
\Omega_{i j}^{\dagger} & T_{j j}
\end{array}\right], \quad \text { with } K=\left[\begin{array}{ll}
k_{i}^{T} & k_{j}^{T}
\end{array}\right]^{T}
$$

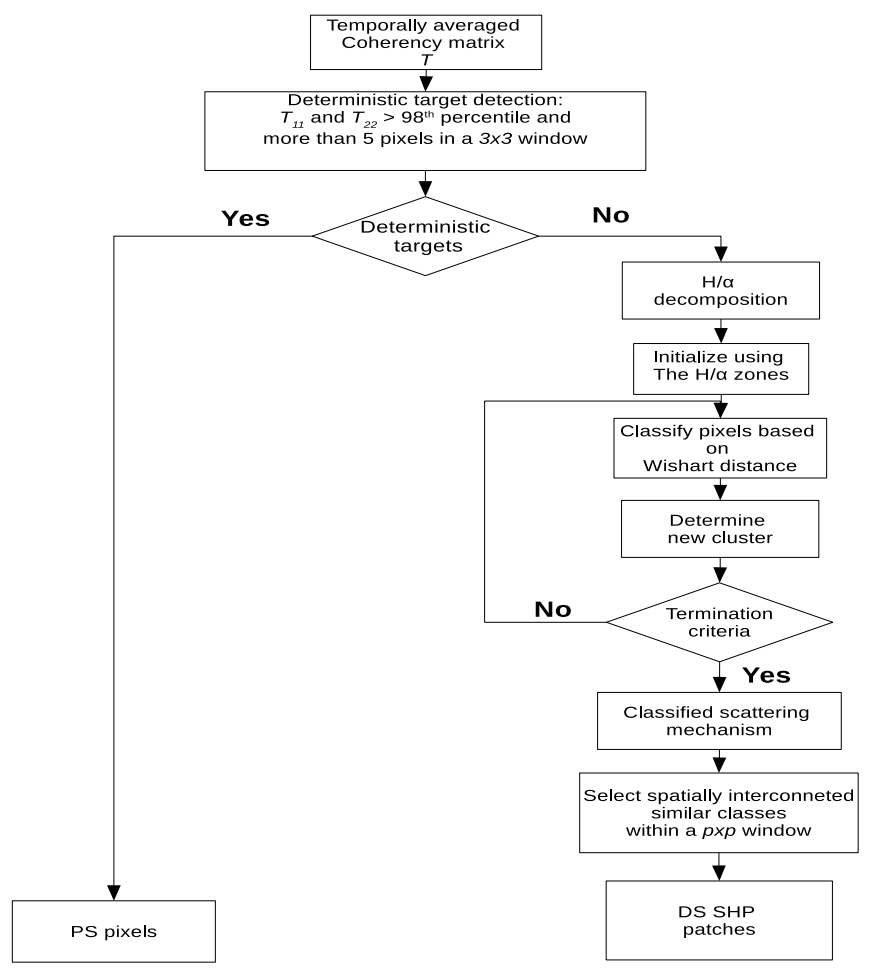

Fig. 1. Methodology flowchart for the selection of PS and DS SHPs.

where $T_{i i}$ and $T_{j j}$ are the coherency matrices related to images $i$ and $j$, respectively, and $\Omega_{i j}(i \neq j)$ is the polarimetric interferometric correlation matrix.

The interferometric coherence $\gamma_{i j}$ is formulated by projecting the scattering vectors $k_{i}$ and $k_{j}$ onto the complex unitary vector $\omega$

$$
\gamma_{i j}=\left|\gamma_{i j}\right| e^{i \phi}=\frac{\omega^{\dagger} \Omega_{i j} \omega}{\sqrt{\left(\omega^{\dagger} T_{i i} \omega\right)\left(\omega^{\dagger} T_{j j} \omega\right)}} .
$$

Coherence optimization aims at finding the $\omega$ values that maximize the average coherence amplitude. The complex unitary projection vector $\omega$ can be represented with parameters that resemble the physical attributes of radar targets, as shown in (6). Hence, it is parameterized as

$$
\omega=\left[\begin{array}{c}
\cos \alpha \\
e^{i \delta} \sin \alpha \cos \beta \\
e^{i \gamma} \sin \alpha \sin \beta
\end{array}\right]
$$

with

$0 \leq \alpha \leq \pi / 2, \quad 0 \leq \beta \leq \pi, \quad-\pi \leq \delta \leq \pi$ and $-\pi \leq \gamma \leq \pi$.

For dual-polarized data, $\omega$ reduces to

$$
\omega=\left[\begin{array}{c}
\cos \alpha \\
e^{i \delta} \sin \alpha
\end{array}\right]
$$

The optimal scattering mechanism $\omega$ is obtained by numerical optimization of the four parameters described in (12) or the two parameters in (13) that provide the highest coherence amplitude in (11). Numerical optimization routines, such as the conjugate gradient descent method [10], can be used to obtain the optimal parameter values. 


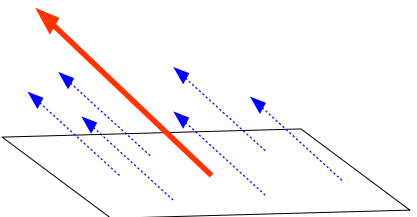

(a)

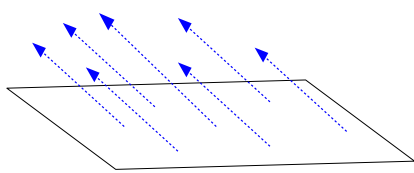

(b)

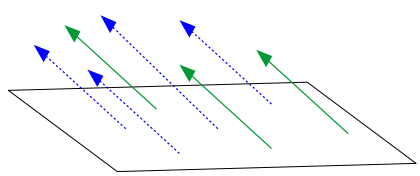

(c)

Fig. 2. Pixel classes utilized in multitemporal InSAR analysis. (a) PS candidate. (b) DS candidate. (c) DS candidate with two distinct scattering types.

\section{E. DS Selection and Processing}

In the traditional PSI analysis, PS candidates are targeted for further analysis in the multitemporal InSAR processing framework. These pixels correspond to a strong point scatterer, where all other secondary scatterers present within the resolution cell are dominated [see Fig. 2(a)]. In contrast, DS candidates are characterized by a scattering mechanism where none of the mechanisms dominate [see Fig. 2(b) and (c)]. In practice, it can either be one type of scattering mechanisms in the resolution cell where none of the mechanisms dominate [see Fig. 2(b)] or it can be a mixture of different scattering mechanisms where some mechanisms are slightly dominant on the others [see Fig. 2(c)]. If there is a mixture of scattering mechanisms within the resolution cell, phase decomposition [2], [11] can be applied to select the most stable scattering mechanisms for application into the different multitemporal InSAR analysis methods [9], [14], [27].

To characterize the differential phase vectors of DS pixels, we first need to derive the interferometric coherence matrix $\Gamma$ [9]. To this effect, we extend the polarimetric optimization routine described in Section II-D from a singlebaseline to a multibaseline case to determine the optimal polarization state $\omega$. The polarimetric interferometric coherency matrix $Z$ discussed in (10) is expanded to the multibaseline case as

$$
Z=\frac{1}{N_{W}} \sum_{K \in \eta} K K^{\dagger}=\left[\begin{array}{ccc}
T_{i i} & \cdots & \Omega_{i n} \\
\vdots & \ddots & \vdots \\
\Omega_{i n}^{\dagger} & \cdots & T_{n n}
\end{array}\right]
$$

where $K=\left[\begin{array}{lll}k_{i}^{T} & \cdots & k_{n}^{T}\end{array}\right]^{T}, \eta$ represents a patch of homogeneous pixels defined in Section II-C containing $N_{W}$ pixels and $i \in[1,2, \ldots, n]$. In the multibaseline case, the optimal polarization state $\omega$ is obtained by numerical optimization of the parameters described in (12) and (13) that provides the highest average coherence of the $((n(n-1)) / 2)$ set of interferograms. Therefore, $\Gamma$ is constructed as

$$
\Gamma=\left\langle s s^{\dagger}\right\rangle=\frac{1}{N_{W}} \sum_{K \in \eta} s s^{\dagger}=\left[\begin{array}{cccc}
1 & \gamma_{12} & \cdots & \gamma_{1 n} \\
\gamma_{12}^{*} & 1 & \cdots & \gamma_{2 n} \\
\vdots & \vdots & \ddots & \vdots \\
\gamma_{1 n}^{*} & \gamma_{2 n}^{*} & \cdots & 1
\end{array}\right]
$$

where $s=\left[\begin{array}{lll}s_{i} & \cdots & s_{n}\end{array}\right]^{T}$ is the normalized polarimetric scattering coefficient of a stack of $n$ images which is derived as $s_{i}=\left(I_{i} /\left(\left(I_{i} I_{i}^{*}\right)^{1 / 2}\right)\right)$ with $I_{i}=\omega^{\dagger} k_{i}$. $\Gamma$ is distinctly different from $T$ and $Z$, because it contains only interferometric information. $\Gamma$ is a Hermitian positive semidefinite matrix where each element corresponds to the complex interferometric coherence $\gamma_{i j}$, with the modulus representing the coherence amplitude and the argument representing the interferometric phase. The flat earth and topographic phase contribution are removed from the off-diagonal components of $\Gamma$ prior to adaptive spatial filtering.

An eigenvalue decomposition routine is applied to decompose $\Gamma$ into its respective eigenvalues and eigenvectors using a singular value decomposition (SVD). It is given as

$$
\Gamma=Q \Lambda Q^{*}=\sum_{i=1}^{n} \lambda_{i} q_{i} q_{i}^{\dagger}
$$

where $Q=\left[q_{1} \ldots q_{n}\right]$ is the orthogonal eigenvectors arranged in columns and $\Lambda$ is the diagonal matrix containing nonzero eigenvalues $(\lambda)$ arranged in the decreasing order. The eigenvectors represent the scattering mechanisms, and the eigenvalues represent the statistical weight assigned to each scattering mechanism.

To describe the statistical disorder of each scatterer type, the scattering entropy $H$ described in Section II-B can be used. We can modify the equation for $H$ to derive the statistical disorder of the phases $(\Psi)$ in a resolution cell as

$\Psi=-\sum_{k=1}^{n} P_{k} \log _{n}\left(P_{k}\right)$ with $P_{i}=\frac{\lambda_{i}}{\sum_{k=1}^{n} \lambda_{k}}$ and $\sum_{k=1}^{n} P_{k}=1$.

For low $\Psi$ values, the resolution cell is dominated by a deterministic point scatterer that dominates other scatterers. Therefore, the second and higher eigenvalues within the resolution cell may be neglected as noise sources. For strong point scatterers, such as PS candidates, the SVD decomposition results in one nonzero eigenvalue representing the strong scatterer, indicating a single dominant scattering mechanism [17]. In this case, (16) simplifies to $\Gamma=\lambda_{1} q_{1} q_{1}^{\dagger}$. Hence, for PS candidates, the coherence matrix obtained from the dominant scattering mechanism $\Gamma(q 1)$ and the original coherence matrix are nearly identical, i.e., $\Gamma \approx \Gamma(q 1)$. In practice, for entropy values closer to zero, the scattering media can be considered as deterministic, and the dominant scattering matrix component can be extracted as the eigenvector corresponding to the largest eigenvalue [3], [4]. In this case, interference of secondary scatterers with the dominant scattering mechanism is insignificant and can be ignored. Hence, the optimal average coherence values can be achieved by discarding all secondary scattering mechanisms, and the optimal coherence matrix $\Gamma(q)$ reduces to $\Gamma(q)=\Gamma(q 1)[2]$.

For DS candidates with higher $\Psi$ values, two cases are distinguished. The first case is that $\Psi$ is close to 1 . This indicates that there are at least $n$ mutually orthogonal scattering mechanisms with a similar amplitude. In this case, scattering can be characterized as a random noise process. Hence, it is considered a random DS and will yield a low-coherence 


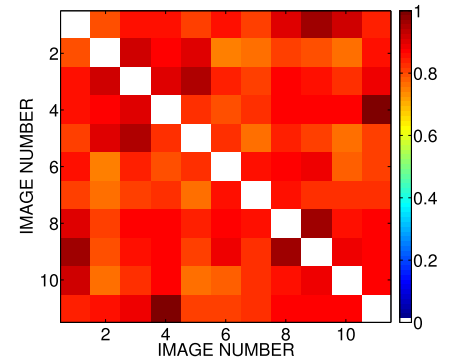

(a)

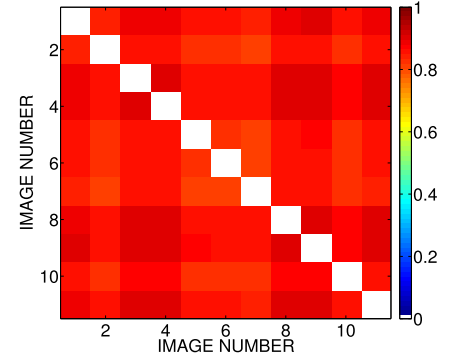

(c)

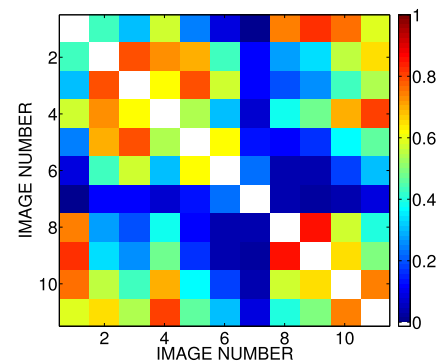

(b)

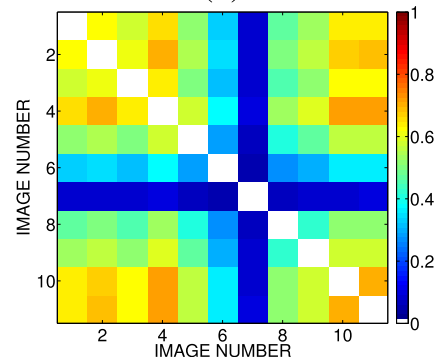

(d)
Fig. 3. Absolute value of the polarimetric interferometric coherence matrix extracted from ALOS PALSAR data. (a) PS candidate for $\Gamma$. (b) DS candidate for $\Gamma$. (c) PS candidate derived from adaptive selection of constructively interfering scattering mechanisms $\Gamma(q)$. (d) DS candidate derived from adaptive selection of constructively interfering scattering mechanisms $\Gamma(q)$. For the PS candidate, the coherence amplitude is high in all interferograms in both $\Gamma$ and $\Gamma(q)$. For the DS candidates [(b) and (d)], it can clearly be seen that coherence improves in almost all interferograms except in images six and seven where the effect of baseline decorrelation occurs.

estimate, and the pixel is useless for multitemporal InSAR analysis. The second case is that $\Psi$ achieves a moderate value. This indicates that there are $n$ dominant scattering mechanisms with decreasing weights from most dominant to least dominant. An analysis of secondary scattering mechanisms and their differential phase interference pattern, however, revealed that the differential phases of some scattering mechanisms are in-phase. The scattering mechanisms are in-phase when they follow the same differential phase pattern indicating the same deformation pattern. Therefore, the coherence matrix adds up to a higher pixel coherence amplitude. Hence, by adaptively selecting only the scattering mechanisms with differential phases that are in-phase with the primary scattering mechanism, we can maximize the amplitude of the coherence sum. Even for very low entropy values, however, some of the secondary scattering mechanisms may interfere constructively, thus providing an incremental improvement to the coherence. However, the coherence from the strong scatterer is already high [see Figs. 3(a) and (c) and 4(a) and (c)], higher than the quality threshold imposed on multitemporal InSAR processing. Hence, for computational efficiency, it is suggested to select a threshold for entropy values where the secondary scattering mechanisms are significant enough to increase the coherence estimate above the threshold values. The selection of these threshold values should be evaluated on a case-tocase basis. The adaptive selection of secondary scattering mechanisms that interfere constructively is achieved by first defining an arbitrary interferometric coherence matrix $\Gamma(C)$

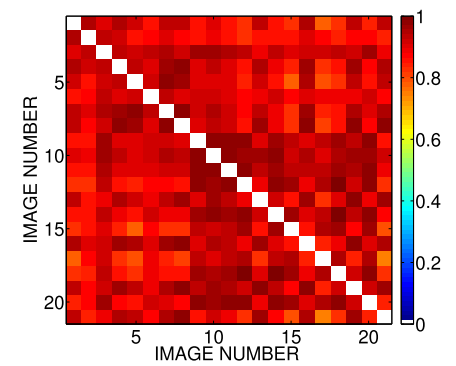

(a)

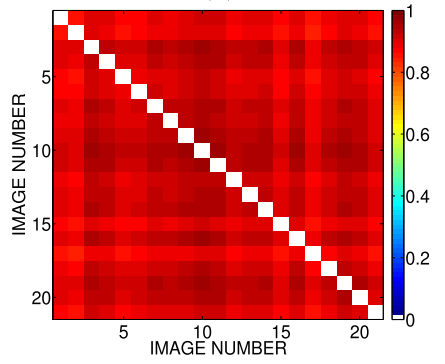

(c)

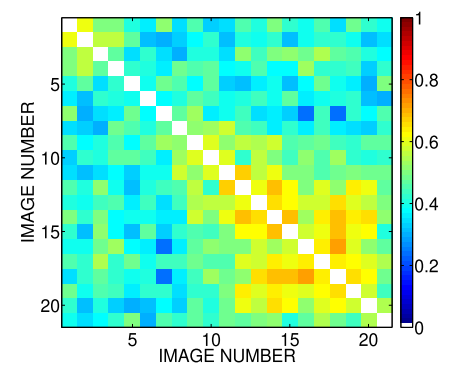

(b)

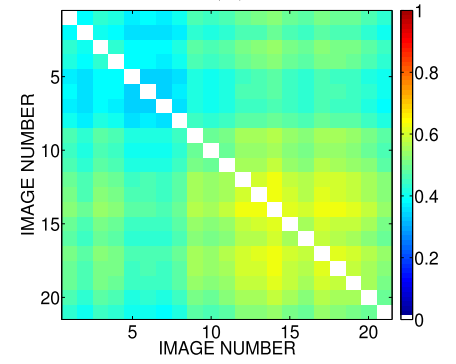

(d)
Fig. 4. Absolute value of the polarimetric interferometric coherence matrix extracted from Sentinel-1 data. (a) PS candidate for $\Gamma$. (b) DS candidate for $\Gamma$. (c) PS candidate derived from adaptive selection of constructively interfering scattering mechanisms $\Gamma(q)$. (d) DS candidate derived from adaptive selection of constructively interfering scattering mechanisms $\Gamma(q)$.

constructed from the subset of eigenvectors as

$$
\Gamma(C)=\sum_{i \in C} \lambda_{i} q_{i} q_{i}^{\dagger} \text { where } C \subset\{1,2, \ldots, n\}
$$

where $C$ is the subset of the eigenvector indices. Since the coherence matrices are Hermitian positive semidefinite matrices of which the strictly upper triangular elements represent unique values for the interferometric phase. We use the $((n(n-1)) / 2)$ strictly upper triangular elements of the coherence matrix in the optimization routine. The optimization criterion for selecting the constructively interfering scattering mechanisms is the amplitude of the sum of complex coherence values given as

$$
\epsilon(C)=\left|\sum_{b=2}^{n} \sum_{a=1}^{b-1}\left[\gamma_{a b}\right]\right|
$$

where $a$ and $b$ are the row and column number of the elements in $\Gamma(C)$ and $\epsilon(C)$ is the amplitude of the sum of the upper traingular coherence values in $\Gamma(C)$. The optimal set of eigenvectors $C_{\text {opt }}$ selected by discarding the unstable scattering mechanisms is given by

$$
C_{\mathrm{opt}}=\arg \max _{C}\{\epsilon(C)\}
$$

and the optimal coherence matrix $\Gamma(q)$ calculated from the selected optimal subset of eigenvectors is given as

$$
\Gamma(q)=\sum_{i \in C_{\mathrm{opt}}} \lambda_{i} q_{i} q_{i}^{\dagger}
$$

As is evident from (20), the amplitude of the coherence sum is maximized by using an exhaustive search optimization. This can be computationally expensive if a large stack of images is being processed. 
TABLE I

List of Sentinel-1 and ALOS PALSAR Data Used In This Paper

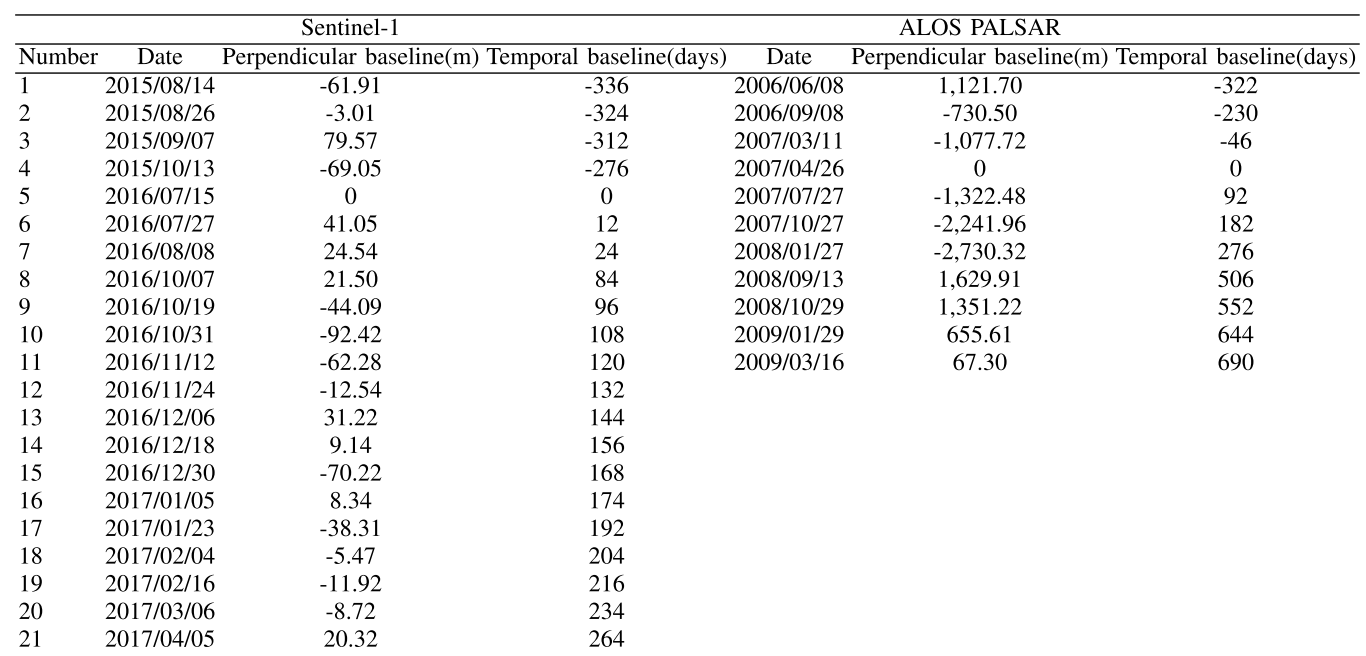

The proposed method workflow is summarized as follows.

1) Apply the scattering property-based adaptive spatial filter to identify and filter DS.

2) Implement coherence optimization to derive the optimal polarization state $\omega$. This is an optional step.

3) Derive the scattering entropy $\Psi$ by applying target decomposition to polarimetric interferometric coherence matrix $\Gamma$ and select the target DS by applying a threshold to $\Psi$.

4) Adaptively select constructively interfering scattering mechanism of the selected DS by maximizing the amplitude of the complex coherence sum in the polarimetric interferometric coherence matrix $\Gamma$.

5) Integrate the optimal coherence matrix $\Gamma(q)$ into the QPS interferometry multitemporal InSAR analysis.

To remove the phase contributions from orbital, digital elevation model (DEM) errors and the atmospheric phase screen and estimate the parameters of interest, i.e., linear velocity and target height we select coherent candidate points by using average coherence derived from $\Gamma(q)$. These coherent points are connected in a spatial network, and numerical integration is performed with respect to a reference point as defined in the QPS processing flow [27]. The QPS technique differs from the PSI technique in three aspects. Interferograms are filtered and are not required to be formed from a single common master image, and the target height and displacement are estimated by selecting a subset of interferograms. In this way, it inserts the coherence value as a weight to make sure that only coherent interferograms in $\Gamma(q)$ influence the result of estimation. Hence, no phase triangulation is required to return to $n-1$ interferograms as in the SqueeSAR [9].

\section{DATA SETS}

To evaluate the performance of the proposed method, 11 quad-polarized ALOS PALSAR images acquired from June 8, 2006 to March 16, 2009 over the San Fernando Valley, CA, USA (see Table I) and 21 dual-polarized Sentinel-1 images acquired from August 14, 2015 to April 5, 2017 over the Groningen area in The Netherlands (see Table I)

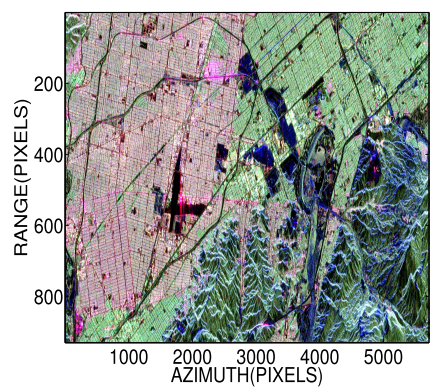

(a)

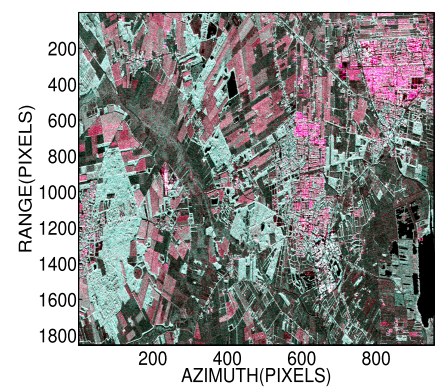

(b)
Fig. 5. Location map of the two test areas. (a) False color composite image obtained by incoherent temporal average of 11 ALOS PALSAR images of San Fernando Valley, CA, USA. Blue region: $\left|S_{\mathrm{HH}}+S_{\mathrm{VV}}\right|^{2}$. Red region: $\mid S_{\mathrm{HH}}-$ $\left.S_{\mathrm{VV}}\right|^{2}$. Green region: $\left|2 S_{\mathrm{VH}}\right|^{2}$. (b) False color composite image obtained by the incoherent temporal average of 21 Sentinel-1 images of the Groningen area, The Netherlands. Blue region: $\left|S_{\mathrm{VV}}+S_{\mathrm{VH}}\right|^{2}$. Green region: $\left|S_{\mathrm{VH}}\right|^{2}$. Red region: $\left|S_{\mathrm{VVV}}\right|^{2}$.

were used. The ALOS PALSAR sensor acquires data in the L-band for the quad-polarization images, whereas the Sentinel-1 sensor acquires data in the C-band for both dual- and single-polarimetric images (see Table II). The test areas contain $930 \times 5715$ pixels and cover $10.76 \mathrm{~km} \times$ $20.23 \mathrm{~km}$ for the ALOS PALSAR and $1855 \times 955$ pixels and $13.3 \mathrm{~km} \times 6.8 \mathrm{~km}$ for the Sentinel-1 data in the range and azimuth directions, respectively. The ALOS PALSAR image consists of predominantly urban region [see Fig. 5(a)], and the Sentnel-1 image consists of a predominantly natural environment that consists of agricultural area [see Fig. 5(b)]. The polarimetric content of the two scenes can be observed from the false color composites of the two regions (see Fig. 5).

\section{RESUlts}

Pixel similarity within a pixel neighborhood is established by implementing the Wishart distance classification method [19]. The average coherency matrix is first constructed by using single-look coherency matrices in the temporal dimension. The scattering property for the scene, i.e., average 


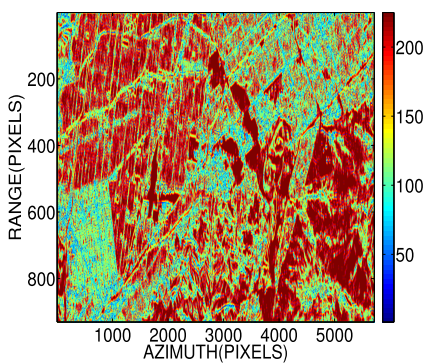

(a)

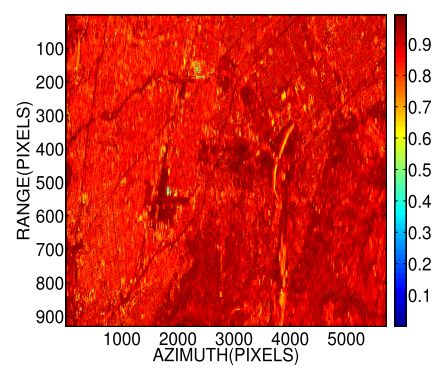

(b)
Fig. 6. (a) Number of homogenous pixels identified within $15 \times 15$ window by the classification of scattering mechanisms for the ALOS PALSAR data. (b) Scattering entropy for the study area. Low entropy values represent a deterministic single dominant scattering mechanism, whereas high entropy values represent multiple random scattering mechanisms within a resolution cell.

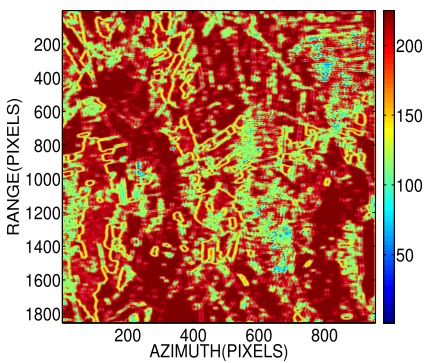

(a)

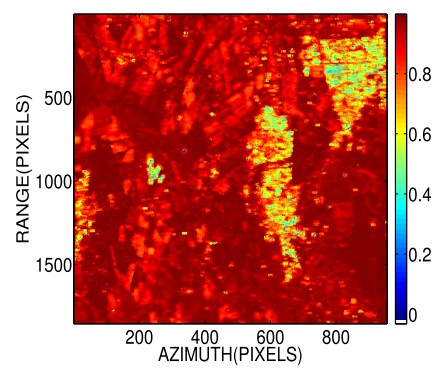

(b)
Fig. 7. (a) Number of homogenous pixels identified within $15 \times 15$ window by the classification of scattering mechanisms from Sentinel-1 data. (b) Scattering entropy for the study area.

\section{TABLE II}

ACQUisition PARAMETERS FOR THE ALOS PALSAR QUAD-POLARIZED IMAGES AND SENTINEL-1 DUAL-POLARIZED IMAGES

\begin{tabular}{lcc}
\hline & Quad polarized data & Dual polarized data \\
\hline Sensor & ALOS-PALSAR & Sentinel-1A and 1B \\
Resolution & $11.38 \mathrm{~m} \times 3.54 \mathrm{~m}$ & $3.67 \mathrm{~m} \times 13.94 \mathrm{~m}$ \\
Incidence angle & $23.6^{0}$ & $39.35^{0}$ \\
Orbit & Ascending & Ascending \\
Temporal baseline & 46 days & 12 days \\
$\begin{array}{l}\text { Dates } \\
\text { Number of images }\end{array}$ & June 8, 2006 - March 16,2009 August 14,2015 - April 05, 2017 \\
\hline
\end{tabular}

scattering mechanism $(\tilde{\alpha})$ and the scattering entropy $(H)$ are derived by implementing the Cloude-Pottier eigenvalueeigenvector decomposition method [5]. $\tilde{\alpha}$ derived in Section II-B by implementing the Cloude-Pottier eigenvalueeigenvector decomposition assumes different values from the optimal scattering mechanism $\alpha$ defined in this section. The reason is that $\tilde{\alpha}$ describes the dominant scattering mechanism present within the resolution cell, whereas $\alpha$ defines the scattering mechanism that maximizes the interferometric coherence in a polarimetric SAR data stack. Hence, $\tilde{\alpha}$ and $\alpha$ have similar values in resolution cells, where there is a deterministic point scatterer. In DS scatterers, $\tilde{\alpha}$ and $\alpha$ assume different values. To implement the Wishart distance classification routine for the classification of scattering properties, $\tilde{\alpha}$ and $H$ values were plotted on the $H / \alpha$ plane, and the center points in each class were selected to initialize the classification process. We implemented the combination of the unsupervised target decomposition classifier and the supervised Wishart classifier routine described in Section II-D, which resulted in results in eight distinct scattering mechanism classes. A $10 \%$ pixel switching threshold is used as termination

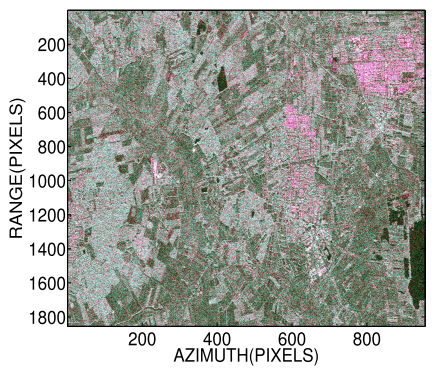

(a)

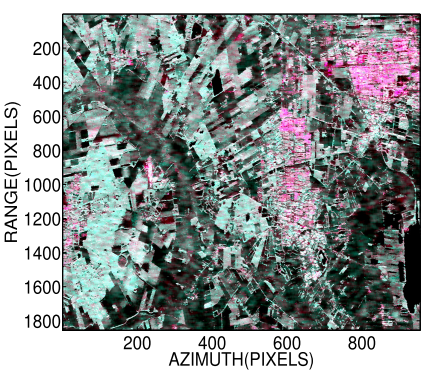

(b)
Fig. 8. Comparison between (a) unfiltered false color composite intensity Sentinel-1 dual-polarization image acquired on August 14, 2015 and (b) false color composite of the same image obtained by applying the scattering property-based adaptive filtering method. Blue region: $\left|S_{\mathrm{VV}}+S_{\mathrm{VH}}\right|^{2}$. Green region: $\left|S_{\mathrm{VH}}\right|^{2}$. Red region: $\left|S_{\mathrm{VV}}\right|^{2}$.

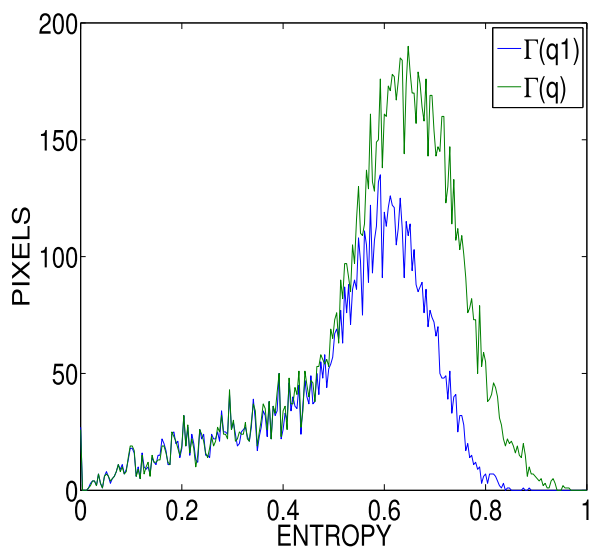

Fig. 9. Comparison of entropy distribution for pixels with temporal coherence greater than 0.9 for the Senitnel- 1 data. For $\Psi>0.4$, selecting the constructively interfering secondary scattering mechanisms with the dominant scattering mechanism $\Gamma(q)$ selects more pixels than by using the dominant scattering mechanism $\Gamma(q 1)$.

criteria. To establish homogeneity within the neighboring of DS pixels, spatial connectivity of pixels of a similar scattering class is assessed. A minimum number of eight pixels within a $15 \times 15$ pixels are used to establish this pixel neighborhood. Pixels that did not fulfill the eight interconnected neighboring pixels were designated as isolated pixels. Isolated pixels not belonging to the PS candidates were merged with the class that has the majority surrounding those pixels. The number of homogenous pixels generated using a $15 \times 15$ pixel neighborhood from the classified scattering class is shown in Figs. 6(a) and 7(a). The effectiveness of the scattering property-based adaptive filtering method is demonstrated by filtering a single-look Sentinel-1 image (see Fig. 8). The scattering property-based adaptive filtering method clearly reduces speckle while preserving feature shapes and edges.

The proposed method adaptively selected the secondary scattering mechanisms that interfere constructively with the dominant scattering mechanisms, hence improving the overall pixel coherence estimate. This can be observed from Figs. 3 and 4. The coherence interferograms with short temporal baselines located near the diagonal line show higher coherence. However, coherence interferograms with long perpendicular baselines [in images 6 and 7 of Fig. 3(b) and (d)] display low-coherence values. With DS decomposition, the coherence values with short temporal baselines show a 
significant increase $(>0.1)$, whereas the coherence interferograms with long spatial and temporal baselines do not show much improvement from the polarimetrically optimized coherence matrix $\Gamma(\omega)$. This is reasonable because with temporal decorrelation, the scattering property of the media changes and may be unrelated to interference of different scattering mechanisms.

We selected the DS candidate points for the application of the proposed method by using the phase entropy $\Psi$ defined in Section II-E. For $\Psi \leq 0.4$, the dominant scattering mechanism $q 1$ achieves the highest coherence value indicating all secondary scattering mechanisms constitute noise. For $0.4<\Psi \leq 0.9$, the proposed method increased the average pixel coherence estimate by removing the noisy scattering mechanism (see Fig. 9).

Results from the proposed method were evaluated in terms of the number of pixels selected as measurement points, i.e., the detail of the derived displacement map and the accuracy of the derived displacement map. We used the dominant scattering mechanism $q 1$ derived from the VV channel $\Gamma(q 1)$ selected by implementing the method detailed in [2] and the proposed method applied on the polarimetrically optimized coherence matrix $\Gamma(\omega)_{q}$ for comparison. For consistency, adaptive spatial filtering in the formation of $\Gamma(\mathrm{VV}), \Gamma(q 1)$, and $\Gamma(\omega)_{q}$ used the same SHP patch. On the ALOS PALSAR data, $\Gamma(\mathrm{VV})$ selected 8091 pixels, $\Gamma(q 1)$ selected 21476 pixels, and $\Gamma(\omega)_{q}$ selected 63605 pixels, respectively (see Table III) by using a temporal coherence threshold $\gamma_{t}>0.9$. To evaluate the improvement obtained when using polarimetric optimization, we compared the number of pixels selected from polarimetrically optimized coherence matrix $\Gamma(\omega)$ and dominant scattering mechanism derived from decomposition of optimized coherence matrix $\Gamma(\omega)_{q 1} . \Gamma(\omega)$ selected 28264 pixels and $\Gamma(\omega)_{q 1}$ selected 42654 pixels.

Next, the proposed method was implemented on a dualpolarized Sentinel-1 data and the VV channel. $\Gamma(\mathrm{VV})$ selected 5244 measurement points, $\Gamma(q 1)$ selected 8639 pixels, and $\Gamma(\omega)_{q}$ selected 13578 pixels, respectively, by using a temporal coherence threshold $\gamma_{t}>0.9$. To evaluate the improvement obtained when using polarimetric optimization, we compared the number of pixels selected from polarimetric optimization, dominant scattering mechanism derived from optimized coherence matrix, and the proposed method. The polarimetrically optimized coherence matrix $\Gamma(\omega)$ selected 8087 pixels, and the dominant scattering mechanism $\Gamma(\omega)_{q 1}$ selected 9976 pixels. The ALOS PALSAR scene is located in a predominantly urban region, where there is a mixture of distinct scattering mechanisms, as shown in Fig. 6(b). Therefore, the proposed method was able to improve the pixel average coherence estimate significantly (see Fig. 10). The Sentinel-1 image, however, is located in a rural region where there was a much more random scattering mechanism with a high entropy [see Fig. 7(b)]. Hence, the proposed method did not provide much improvement in coherence.

The overall computational burden of the proposed method is $6.35 \times 10^{-3} \mathrm{~s}$ per DS candidate pixel. The implementation of the scattering property-based adaptive filtering routine required

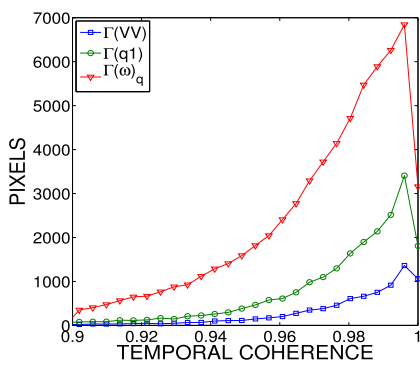

(a) (b)

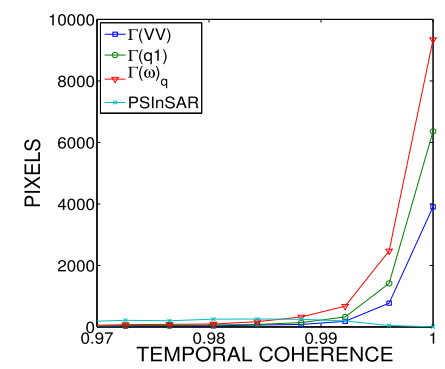

Fig. 10. Temporal coherence derived from (a) ALOS PALSAR data set and (b) Sentinel-1 data set.

\section{TABLE III}

Number of Pixels Selected From the Coherence Matrix Derived FROM VV CHANNEL $\Gamma(\mathrm{VV})$, THE DOMINANT SCATTERING MECHANISM $\Gamma(q 1)$, AND ADAPTIVELY SELECTING THE CONSTRUCTIVELY INTERFERING SCATTERING MECHANISMS FROM THE POLARIMETRICALLY Optimized COHERENCE MATRIX $\Gamma(\omega)_{q}$

\begin{tabular}{lcc}
\hline & ALOS PALSAR & Sentinel-1 \\
\hline PSI & - & $2,234(0.12 \%)$ \\
$\Gamma(V V)$ & $8,091(0.15 \%)$ & $5,244(0.29 \%)$ \\
$\Gamma(q 1)$ & $21,476(0.4 \%)$ & $8,639(0.48 \%)$ \\
$\Gamma(\omega)_{q}$ & $63,605(1.19 \%)$ & $13,578(0.76 \%)$
\end{tabular}

$23 \%$ of the overall computational time per pixel, whereas polarimetric coherence optimization utilizing conjugate gradients required $31 \%$ per pixel. However, the phase entropybased DS identification and the coherence matrix optimization by the selection of constructively interfering scattering mechanism by exhaustive search optimization were computationally expensive, especially if the full set of eigenvectors were considered for optimization. It required $3.42 \times 10^{-3} \mathrm{~s}$ per DS pixel for the ALOS PALSAR data (11 images) and 8.98 s per DS pixel for the Sentinel-1 data (21 images) when implemented on MATLAB run with a Linux operating system on an Intel core i7 $2.70-\mathrm{GHz}$ processor laptop with eight processing cores. It is clear that DS identification and exhaustive search optimization of constructively interfering scattering mechanism are the most expensive one. The computational efficiency is significantly improved by imposing a threshold on the minimum value of eigenvalue for the eigenvector to be considered for optimization. It required $46 \%$ of the overall processing time when a threshold of 0.2 is applied on the eigenvalue when processing the Sentinel-1 data.

The deformation maps provided by the proposed method were compared with the VV channel and the dominant scattering mechanism derived from it. We derived the displacement maps shown in Figs. 11 and 12 using the SARPROZ multitemporal InSAR processing software [28]. The deformation trend estimated from each method showed good agreement. However, the details provided by the proposed method is higher than $\Gamma(\mathrm{VV})$ and $\Gamma(q 1)$. In the ALOS PALSAR scene, more details are provided by the proposed method than by the other methods, thereby clearly delineating the boundaries of the subsidence phenomena [see Fig. 11(c)]. We have not included the results of PSI for the ALOS PALSAR data set, since a minimum number of 15-20 images are required for a PSI analysis [7]. With the availability of more images, the quality of PSI analysis increases. 


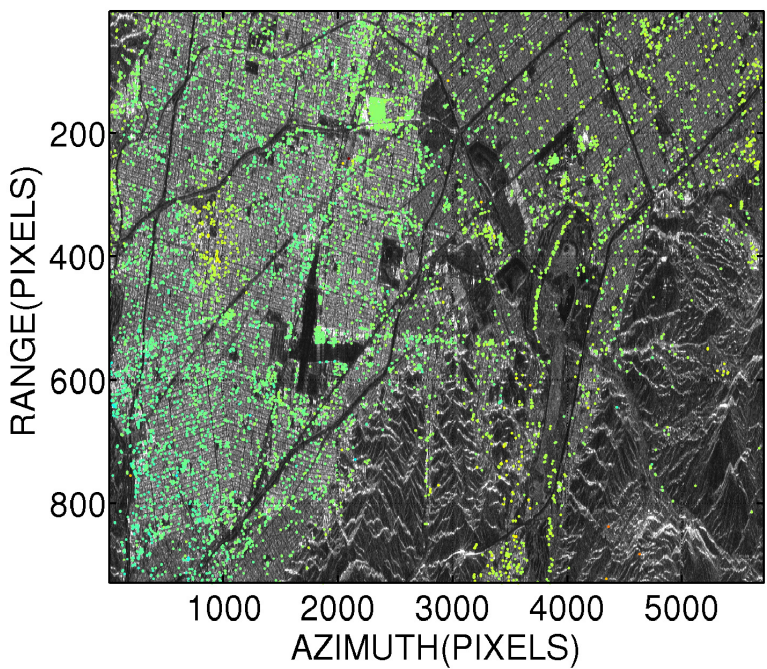

(a)

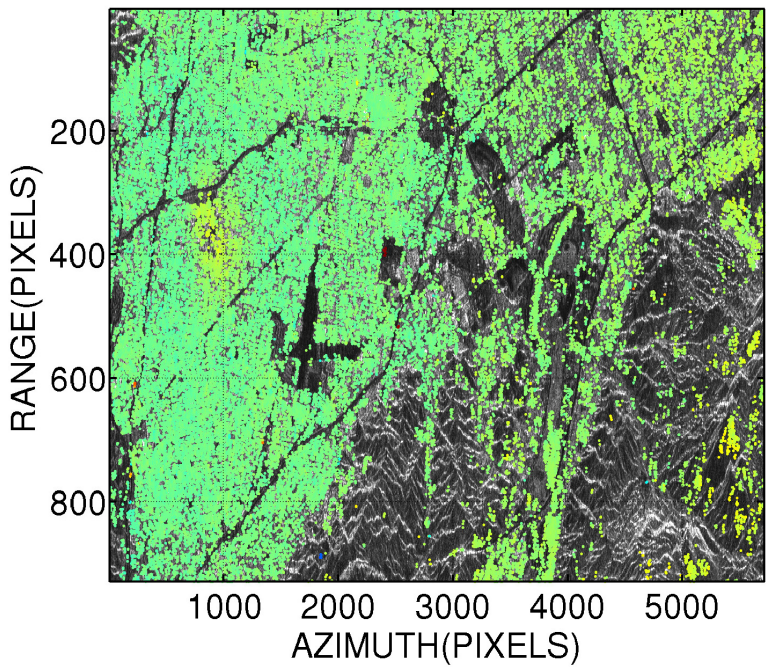

(c)

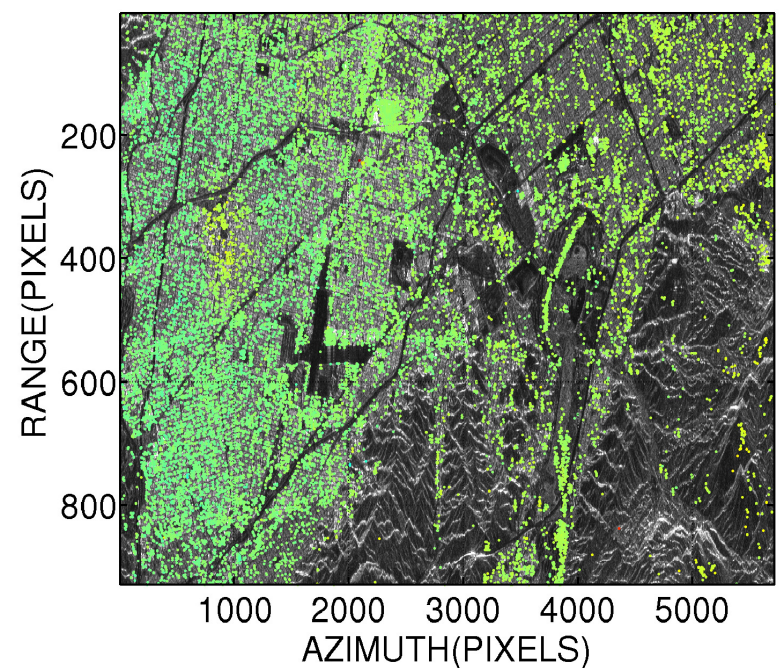

(b)

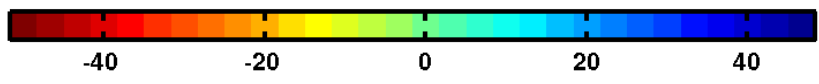

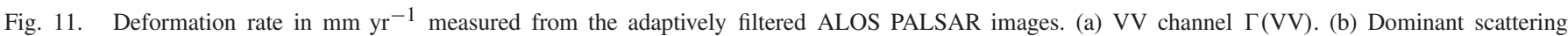
mechanism derived from VV channel $\Gamma(q 1)$. (c) Proposed method $\Gamma(\omega)_{q}$. The PSI method is not included, because the PSI analysis derived from 11 images is unreliable.

To assess the performance of the displacement estimation from the proposed method, we compared the temporal coherence from $\Gamma(\mathrm{VV}), \Gamma(q 1)$, and $\Gamma(\omega)_{q}$ coherence matrices. Temporal coherence is a parameter that measures the phase residual after estimation and removal of atmospheric phase screen, orbital, and DEM phase errors [8]. Higher temporal coherence corresponds with a lower phase residual and a high model fit. As can be seen from Fig. 10, $\Gamma(\omega)_{q}$ provides higher temporal coherence than $\Gamma(\mathrm{VV})$ and $\Gamma(q 1)$. In this way, we conducted a detailed time series analysis around the village of Langelo at a location of a gas extraction plant and the town of Roden for the Sentinel-1 data (see Fig. 13). Fig. 13(a) shows that the VV channel, $\Gamma(q 1)$, and $\Gamma(\omega)_{q}$ provide a similar displacement time series when compared for a PS candidate. However, the accuracy of displacement measurement deviated when comparing a PS candidate and a nearby DS candidate [see Fig. 13(b)]. The reason for this can be the variation in point density, which induces unwrapping errors. This is demonstrated by the time series extracted over the village of Roden [see Fig. 13(a)] with similar point densities, which shows a similar displacement time series for all methods. On the contrary over the Langelo gas extraction site in Groningen [see Fig. 13(b)], the point density is different, and the displacement time series is different due to unwrapping errors. In the future studies, the reliability of each method should be investigated against in situ measurements, such as GPS.

\section{Conclusion And Discussion}

In this paper, the scattering property-based speckle filtering suggested in [20] and [23] for single quad-polarized data has been extended to estimate both quad-polarized and dualpolarized interferometric coherency matrices. Estimation of the polarimetric coherency matrix by averaging in the temporal dimension helped to preserve the scene resolution. This was important in an application to multitemporal InSAR analysis, 


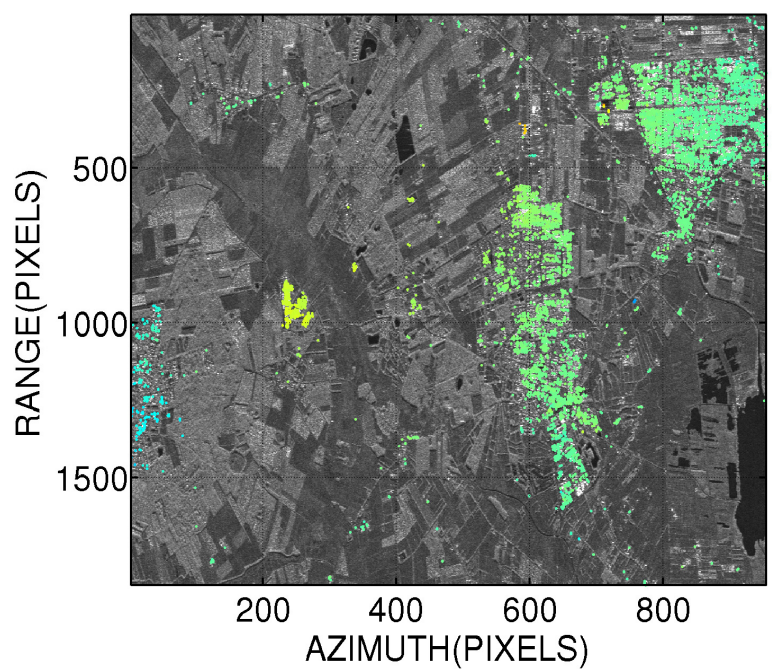

(a)

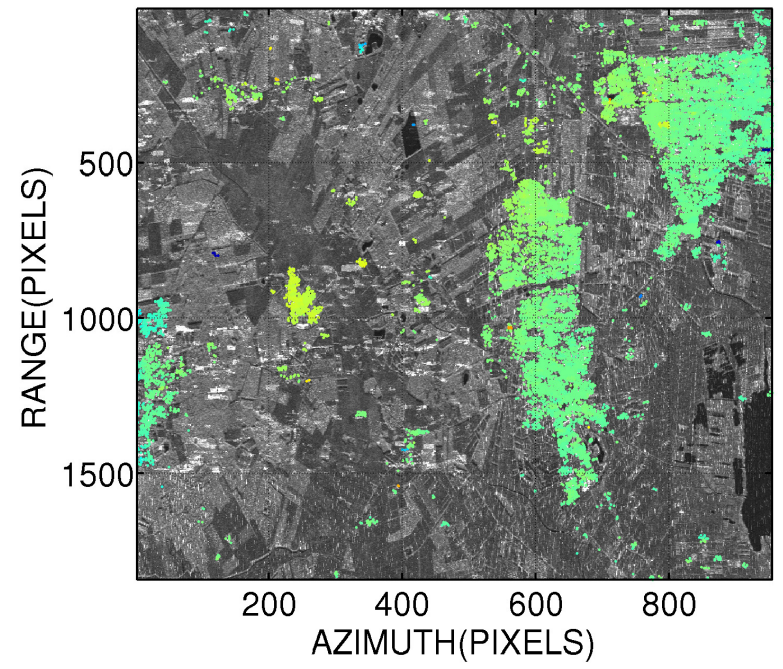

(c)

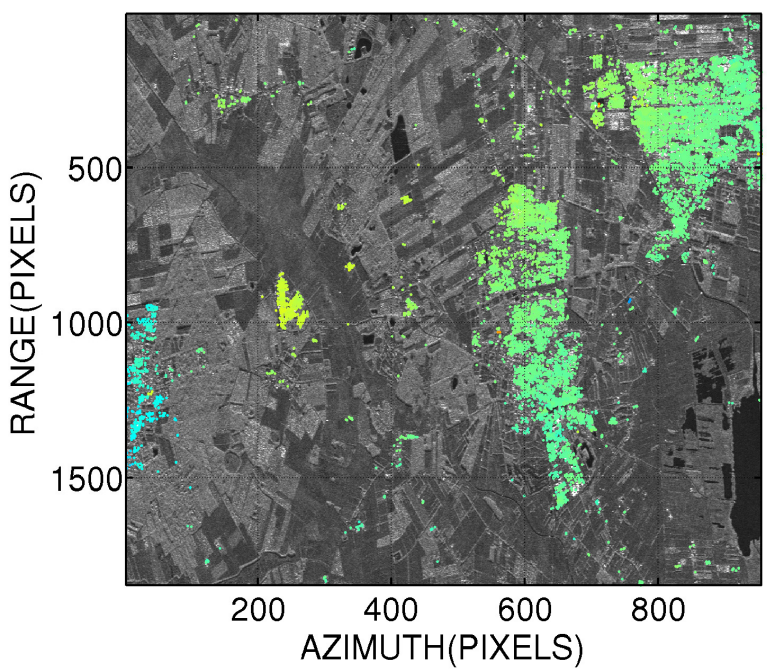

(b)

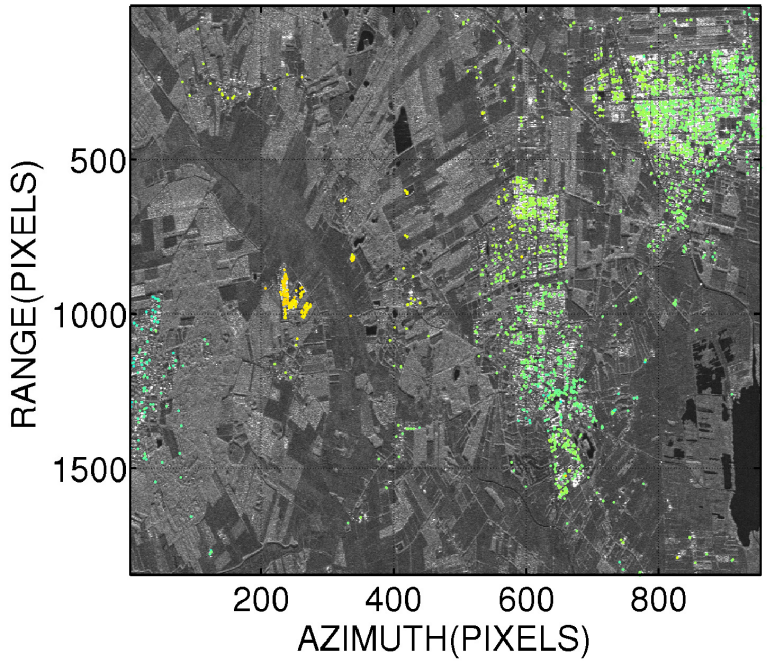

(d)

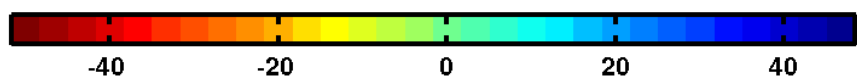

40

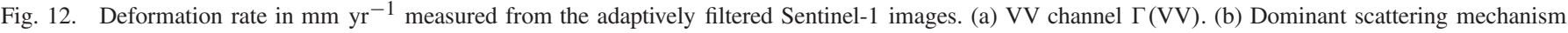
derived from VV channel $\Gamma(q 1)$. (c) Proposed method $\Gamma(\omega)_{q}$. (d) PSI.

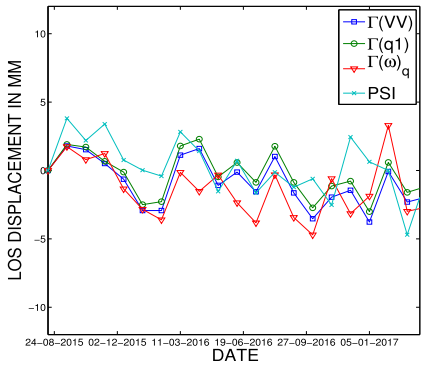

(a)

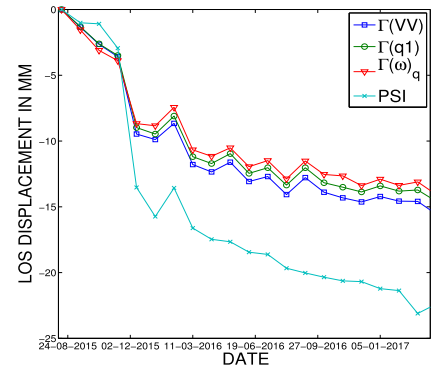

(b)
Fig. 13. Displacement time series obtained from the Sentinel-1 data sets by applying different methods on (a) measurement point from a PS candidate and (b) measurement point taken from a PS pixel candidate and a DS pixel candidate.

where the analysis focuses on pixel candidates that are strongly stable scatterers. The application of adaptive filtering helped to improve the accuracy of coherence estimation, as coherence bias depends upon the number of samples (looks) and the stationarity assumption used during estimation [29]. In this way, maintaining the homogeneity of the neighborhood pixels by applying the scattering property-based adaptive filtering and using a larger averaging window to have a large number of samples minimizes the bias in coherence estimation.

The modified phase decomposition method described in this paper helped improving the interferometric phase quality by discarding noisy scattering mechanisms in the resolution cell. The scattering mechanisms can be understood as scatterers that have distinct phase centers with different height and spatial locations. We exploited the interference pattern of the secondary scattering mechanisms to improve the coherence of the resolution cell. It was effective in removing secondary scattering mechanisms that interfered with other dominant scattering mechanisms. Theoretically, all secondary scattering mechanisms can interfere constructively. In this paper, but only 
a few of those do so, the others constitute noise. Their effects are more pronounced in DS pixels without a significantly dominant scatterer. The proposed method removes these sources of noise from the pixel.

\section{ACKNOWLEDGMENT}

The authors would like to thank the anonymous reviewers for the comments and suggestions, which have improved the quality of this paper.

\section{REFERENCES}

[1] P. Berardino, G. Fornaro, R. Lanari, and E. Sansosti, "A new algorithm for surface deformation monitoring based on small baseline differential SAR interferograms," IEEE Trans. Geosci. Remote Sens., vol. 40, no. 11, pp. 2375-2383, Nov. 2002.

[2] N. Cao, H. Lee, and H. C. Jung, "A phase-decomposition-based PSInSAR processing method," IEEE Trans. Geosci. Remote Sens. vol. 54, no. 2, pp. 1074-1090, Feb. 2016.

[3] S. R. Cloude, "Conditions for the physical realisability of matrix operators in polarimetry," in Proc. 33rd Аnпи. Techn. Symp., 1990, pp. $177-187$.

[4] S. R. Cloude and E. Pottier, "Concept of polarization entropy in optical scattering," Opt. Eng., vol. 34, no. 6, pp. 1599-1610, 1995.

[5] S. R. Cloude and E. Pottier, "An entropy based classification scheme for land applications of polarimetric SAR," IEEE Trans. Geosci. Remote Sens., vol. 35 , no. 1, pp. 68-78, Jan. 1997.

[6] M. Crosetto, E. Biescas, J. Duro, J. Closa, and A. Arnaud, "Generation of advanced ERS and envisat interferometric SAR products using the stable point network technique," Photogram. Eng. Remote Sens., vol. 74, no. 4, pp. 443-450, 2008

[7] M. Crosetto, O. Monserrat, M. Cuevas-González, N. Devanthéry, and B. Crippa, "Persistent scatterer interferometry: A review," ISPRS J. Photogram. Remote Sens., vol. 115, pp. 78-89, May 2016.

[8] A. Ferretti, C. Prati, and F. Rocca, "Permanent scatterers in SAR interferometry," IEEE Trans. Geosci. Remote Sens., vol. 39, no. 1, pp. 8-20, Jan. 2001.

[9] A. Ferretti, A. Fumagalli, F. Novali, C. Prati, F. Rocca, an A. Rucci, "A new algorithm for processing interferometric datastacks: SqueeSAR," IEEE Trans. Geosci. Remote Sens., vol. 49, no. 9, pp. 3460-3470, Sep. 2011.

[10] R. Fletcher and C. M. Reeves, "Function minimization by conjugate gradients," Comput. J., vol. 7, no. 2, pp. 149-154, 1964.

[11] G. Fornaro, S. Verde, D. Reale, and A. Pauciullo, "CAESAR: An approach based on covariance matrix decomposition to improve multibaseline-multitemporal interferometric SAR processing," IEEE Trans. Geosci. Remote Sens., vol. 53, no. 4, pp. 2050-2065, Apr. 2015.

[12] K. Goel and N. Adam, "A distributed scatterer interferometry approach for precision monitoring of known surface deformation phenomena," IEEE Trans. Geosci. Remote Sens., vol. 52, no. 9, pp. 5454-5468, Sep. 2014.

[13] N. R. Goodman, "Statistical analysis based on a certain multivariate complex Gaussian distribution (an introduction)," Ann. Math. Statist., vol. 34, no. 1, pp. 152-177, 1963.

[14] A. Hooper, "A multi-temporal InSAR method incorporating both persistent scatterer and small baseline approaches," Geophys. Res. Lett., vol. 35 , no. $16,2008$.

[15] R. Iglesias, D. Monells, X. Fabregas, J. J. Mallorqui, A. Aguasca, and C. López-Martinez, "Phase quality optimization in polarimetric differential SAR interferometry," IEEE Trans. Geosci. Remote Sens., vol. 52 , no. 5, pp. 2875-2888, May 2014.

[16] K. Ji and Y. Wu, "Scattering mechanism extraction by a modified cloudepottier decomposition for dual polarization SAR," Remote Sens., vol. 7 , no. 6, pp. 7447-7470, 2015.

[17] J.-S. Lee and E. Pottier, Polarimetric Radar Imaging: From Basics to Applications. Boca Raton, FL, USA: CRC Press, 2009.

[18] J.-S. Lee, K. W. Hoppel, S. A. Mango, and A. R. Miller, "Intensity and phase statistics of multilook polarimetric and interferometric SAR imagery," IEEE Trans. Geosci. Remote Sens., vol. 32, no. 5 , pp. 1017-1028, Sep. 1994.

[19] J.-S. Lee, M. R. Grunes, T. L. Ainsworth, L.-J. Du, D. L. Schuler, and S. R. Cloude, "Unsupervised classification using polarimetric decomposition and the complex Wishart classifier," IEEE Trans. Geosci. Remote Sens., vol. 37, no. 5, pp. 2249-2258, Sep. 1999.
[20] J.-S. Lee, M. R. Grunes, D. L. Schuler, E. Pottier, and L. Ferro-Famil, "Scattering-model-based speckle filtering of polarimetric SAR data," IEEE Trans. Geosci. Remote Sens., vol. 44, no. 1, pp. 176-187, Jan. 2006.

[21] J.-S. Lee, J.-H. Wen, T. L. Ainsworth, K.-S. Chen, and A. J. Chen, "Improved sigma filter for speckle filtering of SAR imagery," IEEE Trans. Geosci. Remote Sens., vol. 47, no. 1, pp. 202-213, Jan. 2009.

[22] K.-F. Lin and D. Perissin, "Identification of statistically homogeneous pixels based on one-sample test," Remote Sens., vol. 9, no. 1, p. 37 2017.

[23] A. G. Mullissa, V. Tolpekin, and A. Stein, "Scattering property based contextual PolSAR speckle filter," Int. J. Appl. Earth Observ. Geoinf., vol. 63, pp. 78-89, Dec. 2017

[24] A. G. Mullissa, V. Tolpekin, A. Stein, and D. Perissin, "Polarimetric differential SAR interferometry in an arid natural environment," Int. J. Appl. Earth Observ. Geoinf., vol. 59, pp. 9-18, Jul. 2017.

[25] V. D. Navarro-Sanchez and J. M. Lopez-Sanchez, "Spatial adaptive speckle filtering driven by temporal polarimetric statistics and its application to PSI," IEEE Trans. Geosci. Remote Sens., vol. 52, no. 8, pp. 4548-4557, Aug. 2014.

[26] V. D. Navarro-Sanchez, J. M. Lopez-Sanchez, and L. Ferro-Famil, "Polarimetric approaches for persistent scatterers interferometry," IEEE Trans. Geosci. Remote Sens., vol. 52, no. 3, pp. 1667-1676, Mar. 2014

[27] D. Perissin and T. Wang, "Repeat-pass SAR interferometry with partially coherent targets," IEEE Trans. Geosci. Remote Sens., vol. 50, no. 1 pp. 271-280, Jan. 2012.

[28] D. Perissin, Z. Wang, and T. Wang, "The SARPROZ InSAR tool for urban subsidence/manmade structure stability monitoring in China," in Proc. ISRSE, Sidney, NSW, Australia, 2011, p. 1015.

[29] R. Touzi, A. Lopes, J. Bruniquel, and P. W. Vachon, "Coherence estimation for SAR imagery," IEEE Trans. Geosci. Remote Sens., vol. 37, no. 1, pp. 135-149, Jan. 1999

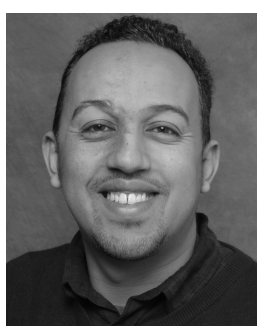

Adugna G. Mullissa received the M.Sc. degree in remote sensing for earth science applications from Addis Abeba University, Addis Abeba, Ethiopia, and the Ph.D. degree in radar remote sensing from the University of Twente, Enschede, The Netherlands.

$\mathrm{He}$ is currently a Researcher with the Department of Earth Observation Science, Faculty of GeoInformation Science and Earth Observation (ITC), University of Twente. His research interests include multitemporal polarimetric differential synthetic aperture radar (SAR) interferometry, pattern recognition, and machine learning for polarimetric SAR data

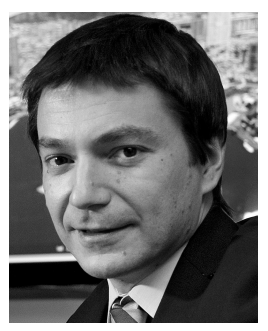

Daniele Perissin was born in Milan, Italy, in 1977. He received the master's degree (Laurea) in telecommunications engineering and the Ph.D. degree (cum laude) in information technology from the Politecnico di Milano, Milan, in 2002 and 2006, respectively.

He joined the Signal Processing Research Group, Politecnico di Milano, in 2002. Since 2002, he has been involved in time-series interferometric synthetic aperture radar analysis in the framework of radar remote sensing. In 2009, he moved to the Institute of Space and Earth Information Science, The Chinese University of Hong Kong, Hong Kong, as a Research Assistant Professor. Since 2013, he has been holding an assistant professor position at the School of Civil Engineering, Purdue University, West Lafayette, IN, USA. He is the Developer of the software Sarproz for processing multitemporal interferometric synthetic aperture radar data. He has authored over 160 research works in journals and conference proceedings. He holds two patents on synthetic aperture radar processing algorithms.

Dr. Perissin received the JSTARS Best Paper Award in 2012 


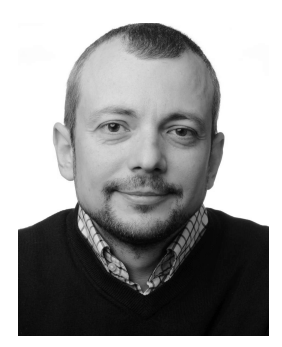

Valentyn A. Tolpekin (M'08) received the M.Sc. degree in physics from Odessa State University, Odessa, Ukraine, and the Ph.D. degree in physics from the University of Twente, Enschede, The Netherlands.

$\mathrm{He}$ is currently an Assistant Professor with the Department of Earth Observation Science, Faculty of Geo-Information Science and Earth Observation (ITC), University of Twente. His research interests include the statistical analysis of optical and synthetic aperture radar images.

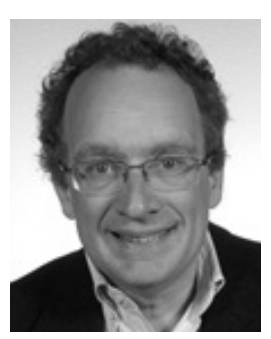

Alfred Stein received the M.Sc. degree in mathematics and information science, with a specialization in applied statistics, from the Eindhoven University of Technology, Eindhoven, The Netherlands, and the $\mathrm{Ph} . \mathrm{D}$. degree in spatial statistics from Wageningen University, Wageningen, The Netherlands.

$\mathrm{He}$ has honorary positions at the University of Pretoria, Pretoria, South Africa, and the University of Cape Town, Cape Town, South Africa. He is currently a Professor of spatial statistics and image analysis with the Faculty of Geo-Information Science and Earth Observation (ITC), University of Twente, Enschede, The Netherlands. He supervises 11 Ph.D. students. His research interests include the statistical aspects of spatial and spatiotemporal data in the widest sense, such as monitoring data, optimal sampling, image analysis, spatial statistics, the use of prior information, the issues of data quality, fuzzy techniques, and random sets in a Bayesian setting, and hazards and disasters, spatial geohealth, and spatial epidemiology.

Dr. Stein has been the Editor-in-Chief of the Spatial Statistics journal, which is the new leading platform in the field of spatial statistics, since 2011. 